\title{
Low-order parabolic theory for 2D boundary-layer stability
}

\author{
Rama Govindarajan \\ National Aerospace Laboratories, Bangalore 560 017, India and Fluid Dynamics Unit, Jawaharlal Nehru \\ Centre for Advanced Scientific Research, Bangalore 560 064, India \\ R. Narasimha \\ Fluid Dynamics Unit, Jawaharlal Nehru Centre for Advanced Scientific Research, Bangalore 560 064, India
}

(Received 2 April 1998; accepted 9 February 1999)

\begin{abstract}
We formulate here a lowest order parabolic (LOP) theory for investigating the stability of two-dimensional spatially developing boundary layer flows. Adopting a transformation earlier proposed by the authors, and including terms of order $R^{-2 / 3}$ where $R$ is the local boundary-layer thickness Reynolds number, we derive a minimal composite equation that contains only those terms necessary to describe the dynamics of the disturbance velocity field in the bulk of the flow as well as in the critical and wall layers. This equation completes a hierarchy of three equations, with an ordinary differential equation correct to $R^{-1 / 2}$ (similar to but different from the Orr-Sommerfeld) at one end, and a "full" nonparallel equation nominally correct to $R^{-1}$ at the other (although the latter can legitimately claim higher accuracy only when the mean flow in the boundary layer is computed using higher order theory). The LOP equation is shown to give results close to the full nonparallel theory, and is the highest-order stability theory that is justifiable with the lowest-order mean velocity profiles for the boundary layer. (C) 1999 American Institute of Physics.
\end{abstract}

[S1070-6631(99)01006-5]

\section{INTRODUCTION}

The downstream growth of a boundary layer of characteristic thickness $\theta(x)$ can be written as

$$
\frac{d \theta}{d x} \sim O\left(\frac{1}{R}\right)
$$

where A- is the streamwise coordinate and $R$ the Reynolds number based on boundary-layer thickness. At high Reynolds numbers, this variation with $x$ is small in comparison with variations of other physical quantities like the velocity with respect to the normal direction $y$. It has therefore been long considered a good first approximation to take the boundary layer as locally parallel in stability analyses, and apply the traditional Orr-Sommerfeld (OS) equation. Several penetrating studies (e.g., Gaster, ${ }^{1}$ Bertolotti et al. $^{2}$ ) sought to improve on the parallel-flow approximation by taking account of the spatial development. In most of these, the OS equation was accepted as being in some sense the lowestorder stability equation, with nonparallel effects adding higher-order $\left[O\left(R^{-1}\right)\right]$ corrections to it. To lend strength to this line of thought, several nonparallel formulations derived from first principles, such as Bertolotti, Herbert, and Spalart ${ }^{2}$ (hereafter BHS) and Govindarajan and Narasimha ${ }^{3}$ (hereafter GN95), gave rise to more elaborate stability equations which contain the entire OS equation and several additional terms with $R^{-1}$ as factor. Some of these higher-order terms contain derivatives in the streamwise direction, which means that the stability equations are no longer ordinary differential equations like the Orr-Sommerfeld. Although the apparent difference between the parallel and nonparallel formulations is only in the higher-order terms, the implicit assumption that the stability of a boundary layer is not strongly affected by its growth was (for various reasons discussed below) in need of a critical re-examination, which has recently been undertaken by Govindarajan and Narasimha ${ }^{4}$ (hereafter GN97). Considering similarity solutions of the boundary-layer equations, and adopting a coordinate transformation inspired by the similarity variables, they showed that the spatial development of the flow affects stability at an order considerably lower than $O\left(R^{-1}\right)$.This does not present any contradiction, since nonparallel effects are contained, not merely in the additional higher-order terms emerging from various formulations, but very substantially in the lower-order terms themselves. They showed that a "lowest-order" stability equation, which contained all effects of $O\left(R^{-1 / 2}\right)$ [and up to but not including $O\left(R^{-2 / 3}\right)$ ], produces stability results in good qualitative agreement with the results of the full nonparallel analysis. This equation is nonetheless an ordinary differential equation; the streamwise dependence is partly explicit, since $R=R(x)$, but is also imbedded into it by the particular coordinate transformation used.

In nonparallel stability analyses which include terms of $O\left(R^{-1}\right)$, the streamwise derivative of the disturbance amplitude $\partial \phi / \partial x$ imparts a parabolic character to disturbance propagation (BHS interpret the presence of this term as the defining feature of nonparallel stability theories). They therefore call their equation the Parabolic Stability Equation (PSE as it is popularly known) and solve it by marching downstream. It is relevant to point out here that in their respective formulations, both BHS and GN95 make the assumption that, since the boundary layer growth rate is $O\left(R^{-1}\right)$, downstream variations in $\phi$ are at most of this order, i.e., 


$$
\frac{i \phi}{f \prime 1}-O\left(R^{-1}\right)
$$

the second derivative $f l^{2} 6 l d x \sim$ being of higher order and therefore negligible. The parabolic effects are therefore presumed to be "weak." The question we pose in this paper is, how important really are the parabolic effects, i.e., given Eq. (2), do they affect stability at a lower order than is apparent at first? If this is indeed the case, is it possible to isolate their "largest" contribution? We proceed to do so by formulating a "lowest-order parabolic" (LOP) stability equation. This equation contains all effects upto and including $O\left(R^{-2 / 3}\right)$.It is shown that stability results obtained from the LOP equation are close to those from a more extended nonparallel analysis of the PSE type.

The present approach places great stress on achieving a rational approximation, i.e., all effects up to a given order in the reciprocal of the Reynolds number are included, and higher-order effects are neglected. Second, compared to BHS, the streamwise derivative is interpreted somewhat differently, leading to different solution methods. The present lowest-order parabolic equation is a subset of the "full" nonparallel equation of GN95, which includes all terms of $O\left(R^{-1}\right)$ in an appropriate primitive equation for the disturbance stream function; since the equation of BHS also includes all terms of this order in addition to others, a comparison between these two equations (GN95 and BHS) brings out the differences in the approaches. The two equations are written out in full and compared at length in Sec. II; for clarity of discussion we introduce here their structure alone. The full nonparallel equation of GN95 has the form

$$
\{\mathrm{OS}\} \phi+\frac{1}{\Omega^{n}}\left\{N P_{1}+N l_{h}\right\} \phi=0\left\{\frac{1}{R^{2}}\right] .
$$

with the Orr-Sommerfeld operator $\{\mathrm{OS}\}$ containing certain terms of $O(1)$ and others with a factor $R^{-1}$. The operator $\left\{N P_{1}\right\}$ consists of nonparallel terms due to the change in the boundary-layer thickness, streamwise variations in the freestream velocity as well as the $x$-dependence of the disturbance. The operator $\left\{N P_{h}\right\}$ accounts for higher-order corrections to the mean flow, due to displacement thickness, surface curvature, etc. (the effect of displacement thickness on the mean flow for Falkner-Skan wedge flows was considered by GN95). Equation (3) includes all terms with the factor $R^{-1}$ in the primitive variables, and will be termed "nominally" correct to $O\left(R^{-1}\right)$ in the following. The PSE, on the other hand, which may be written in the present notation as

$$
\{0 S\} \phi+\frac{1}{R}\left\{N P_{1}\right\} \phi+\frac{1}{R^{2}}\left\{N P_{2}\right\} \phi=O\left[\frac{1}{R^{3}}\right],
$$

contains certain terms of a higher order $\left(R^{-2}\right)$ but omits others of the same order when written out in the variables of GN95. A more consistent version of the PSE has been derived by Simen et al. ${ }^{5}$ in which the higher-order terms are dropped. Since BHS derive their equation for a semi-infinite flat plate, for which $O\left(R^{-1}\right)$ mean flow effects happen to vanish, no operator like $\left\{N P_{h}\right\}$ appears in their equation, but of course in more general flows the operator $\left\{N P_{h}\right\}$ cannot be ignored.

A major qualitative difference between these equations and the Orr-Sommerfeld equation,

$$
\{0 S\} \phi=0,
$$

is that while Eq. (5) is an ordinary differential equation in $y$, the "nonparallel" equations (3) and (4) contain derivatives with respect to $x$ as well. The PSE, as mentioned before, is solved by space marching. GN95, on the other hand, noting that $\partial \phi / \partial x$ is independent of the streamwise coordinate $x$ to the order considered, treat it as a perturbation on an ordinary differential equation, and solve Eq. (3) by a trial and error procedure. In the case of a boundary layer over a semiinfinite flat plate, the two methods when applied to the same equation lead to virtually identical solutions; details of the differences in the equations and approaches are discussed in the following sections.

The LOP theory assumes importance in the light of the following discussion. The mean flow contains contributions of $O\left(R^{-1}\right)$ except in the special case of flow over an idcal semi-infinite flat plate. A stability analysis conducted using a full nonparallel equation including all terms of $O\left(R^{1}\right)$ would be rational only if the mean flow were correct up to this order. Apart from it being not always feasible for the mean flow to be prescribed to this degree of accuracy, it is obvious that nonparallel effects must exist even when only the lowest-order contributions to the mean flow are considered. However, the OS does not arise as the correct equation in any rational approximation of the full boundary-layer stability equation: the equation correct to the lowest order is that formulated in GN97. A rational stability theory (LOP) correct to the next higher order is formulated in this paper and it is shown that the LOP is the highest-order theory consistent with a low-order mean flow. Since most stability analyses are conducted using low-order mean How, the LOP, being considerably simpler than the PSE, is of practical uliiity.

The suggestion implicit in the above statements is thut the spatial development of the flow affects stability al a lower order than $O\left(R^{-1}\right)$. A rational stability theory considering all effects including $R^{-1 / 2}$ was formulated by GN97; with neutral stability defined correctly for the flow quantity being studied, this theory was shown to contain most nonparallel effects. Up to this order, there is no explicit effect of the downstream propagation of the disturbances on the stability. Indeed, a legitimate question about a theory of this type is the following: if an ordinary differential equation in $v$ [like Eq. (5)] has a solution $\phi(y)$, an arbitrary function of $x$ times $\phi(y)$ is also a solution; so how does the $x$-dependence get determined? In practice this question has been answercel, e.g., in $e^{n}$-type calculations, by noting that an o.d.e. like the Orr-Sommerfeld equation, through the dependence of $R$ on $x$, carries $x$ as a parameter. Thus the amplitude of the disturbance at any station is determined by the amplification or attenuation that it suffers through the stability characteristics (computed assuming parallel flow) at the immediately preceding station. A more satisfactory answer to this question 
must, however, proceed from a primitive equation in which the $x$-dependence is explicit; it is clear that the weak dependence on $x$, i.e., the fact that $\partial \phi / \partial x$ is a higher-order term, making the parabolicity singular in some sense, holds the key to the answer. We will pursue this question elsewhere, but confine ourselves in the present paper to showing that parabolic effects first appear at order $R^{-2 / 3}$, and that the solution up to this order is closer to that of the more elaborate nonparallel equations than the solution of the lowestorder ordinary differential equation studied in GN97.

It is relevant to mention here that the present theory is quite different from that of Smith. ${ }^{6}$ The latter work also proposes a rational theory for the nonparallel stability of boundary layers, but its region of validity is much narrower than that of the present theory. First, it is valid only close to the lower limb of the neutral stability boundary where the boundary layer displays a triple deck structure: a lower deck, containing both the critical and the wall layers, an upper deck, where the basic flow is virtually the free stream, and a main deck between the two. Second, the scaling is based on the observation that at very high Reynolds numbers, the neutral wave number on the lower limb varies proportionally with $R^{-1 / 4}$, which holds only for $R \gg R_{\mathrm{cr}}$. The theory of Smith can thus predict neither the critical Reynolds number nor the upper branch (although a five deck theory can be formulated for the asymptotic part of the upper branch). The present theory considers separately the critical layer, the wall layer, and the rest of the boundary layer. It is valid whether or not these layers overlap with each other: e.g., the fact that, close to the lower limb, the wall layer is contained in the critical layer presents no problem and requires no separate treatment. The present theory is therefore not restricted to the neighborhood of either limb of the stability boundary. Another advantage the LOP enjoys over the approach of Smith is that since no asymptotic scaling is assumed, it is valid for $R \gg 1$, which includes the neighborhood of the critical Reynolds number. The present theory is thus uniformly valid over the entire domain in the stability diagram; the price paid is that the equation derived is more complicated than Smith's.

\section{THE BASIC EQUATIONS}

The full nonparallel equation of GN95 is given by Eq. (3) with the boundary conditions

$$
\phi=D \phi=0 \text { at } y=0
$$

and

$$
\phi \rightarrow 0, D \phi \rightarrow 0 \text { as } y \rightarrow \infty
$$

in which the operators $\{\mathrm{OS}\},\left\{N P_{1}\right\}$ and $\left\{N P_{B}\right\}$, respectively, are

$$
\begin{aligned}
\{\mathrm{OS}\}= & \left\{i\left(\omega-\alpha \Phi_{0}^{\prime}\right)\left(D^{2}-\alpha^{2}\right)+i \alpha \Phi_{0}^{m}\right. \\
& \left.+\frac{1}{R}\left\{D^{4}-2 \alpha^{2} D^{2}+\alpha^{4}\right)\right\}
\end{aligned}
$$

$$
\begin{aligned}
\left\{N P_{1}\right\}= & \left\{p \Phi_{0} D^{3}+(2 q-p) \Phi_{0}^{\prime} D^{2}+\left[2 y q \alpha\left(\omega-\alpha \Phi_{0}^{\prime}\right)\right.\right. \\
& \left.-p \alpha^{2} \Phi_{0}+(2 q-p) \Phi_{0}^{\prime \prime}\right] D+\left[(q-2 p) \alpha \omega+p \Phi_{0}^{\prime \prime}\right. \\
& \left.+3(p-q) \alpha^{2} \Phi_{0}^{\prime}\right]+\left(-\omega+3 \alpha \Phi_{0}^{\prime}\right) R \alpha^{\prime} \\
& \left.+\left[\Phi_{0}^{\prime \prime}+3 \alpha^{2} \Phi_{0}^{\prime}-2 \alpha \omega-\Phi_{0}^{\prime} D^{2}\right] R \frac{\partial}{\partial x}\right\}
\end{aligned}
$$

and

$$
\left\{N P_{h}\right\} \equiv-i \alpha\left[\Phi_{1}^{\prime}\left(D^{2}-\alpha^{2}\right)-\Phi_{1}^{\prime \prime \prime}\right]
$$

Here the momentum thickness $d$ and the local free-stream velocity $U$ have been used as scales, with $R=\theta U / \nu, v$ being the kinematic viscosity. The operator $D$ stands for differentiation with respect to $y, y$ being proportional to the FalknerSkan similarity variable and $x$ nondimensionalized in a special way:

$$
y_{d}=\theta y \text { and } d x_{d}=\theta d x
$$

where subscript $d$ stands for the dimensional variable. $\Phi$ and $\phi$ are, respectively, the mean and the disturbance amplitude of the streamfunction $\psi$ :

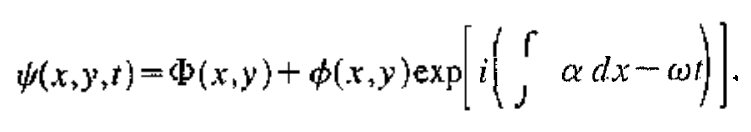

The mean flow contains both lower- and higher-order contributions:

$$
\Phi=\Phi_{0}(y)+\frac{1}{R} \Phi_{1}\left(x_{y} y\right)
$$

(For the particular case considered in GN95, it turns out that $\Phi_{1}$ is independent of $x_{\text {. }}$ ) For a Falkner-Skan velocity profile, the parameters $p$ and $q$, defined, respectively, by the relations

$$
\frac{p U}{R}=\frac{d(U \theta)}{d x_{d}} \text { and } \frac{q}{R}=\frac{d \theta}{d x_{d}}
$$

are constants. Following the same line of reasoning that was used for the assumption (2),

$$
\frac{d^{2} \alpha}{d x^{2}} \sim o\left[R^{-1}\right]
$$

is neglected. The detailed derivation of Eq. (3) is available in GN95.

BHS derive the following equation for the flow over a flat plate under the assumptions (2) and (I5):

$$
\{\mathrm{OS}\} \phi+\left\{\mathcal{L}_{1}\right\} \phi+\left\{\mathcal{L}_{2}\right\} \frac{\partial \phi}{\partial x}+\frac{d a}{d x}\left\{\mathcal{L}_{3}\right\} \phi=0
$$




$$
\begin{aligned}
\left\{\mathcal{L}_{1}\right\}= & -v_{0}\left(\hat{D}^{3}-\alpha^{2} \hat{D}\right)+\hat{D}^{2} v_{0} \hat{D} \\
\left\{\mathcal{L}_{2}\right\}= & -2 \alpha \omega+3 u_{0} \alpha^{2}+\hat{D}^{2} u_{0}-\frac{4 i \alpha^{3}}{R_{0}} \\
& +\left[-u_{0}+\frac{4 i \alpha}{R_{0}}\right] \hat{D}^{2} \\
\left\{\mathcal{L}_{3}\right\}= & -\omega+3 \alpha u_{0}+\frac{2 i}{R_{0}} \hat{D}^{2}-\frac{6 i \alpha^{2}}{R_{0}}
\end{aligned}
$$

and $u_{0}$ and $v_{0}$ are the streamwise and normal components of the mean velocity, respectively, and $\hat{D}$ stands for derivative with respect to the Cartesian coordinate normal to the wall. When it is rewritten in the present notation, the PSE (16) is given by Eq. (4), with

$$
\begin{aligned}
\left\{N P_{2}\right\} \equiv & 2 i q \alpha\left[2 y D^{3}+3 D^{2}-2 y \alpha^{2} D-\alpha^{2}\right] \\
& -4 i \alpha\left(D^{2}-\alpha^{2}\right) R \frac{\partial}{\partial x}-2 i R \alpha^{\prime}\left(D^{2}-3 \alpha^{2}\right) .
\end{aligned}
$$

Since Eq. (4) is written for a Blasius mean flow, the operator $\left\{N P_{1}\right\}$ must be simplified using the fact that $p=q$ in this case for a proper comparison. When this is done it will be seen that Eq. (4) includes all terms of $O\left(R^{-1}\right)$ in Eq. (3), but contains in addition several higher-order terms. [The primitive equations underlying the full nonparallel theory of GN95, however, are the same as the PSE; the higher-order terms in Eq. (17) separate out because of the use of similarity variables in GN95. The approach of GN95 has the advantage that it illuminates connections with Orr-Sommerfeld and similar ordinary differential equations.] It may further be noted that the solutions of GN95 agree well with those of BHS, which shows that the higher-order terms in Eq. (17) are unnecessary, and may be omitted, as expected, without any loss of accuracy.

GN97 formulated the lowest-order stability equation which contains all effects up to and including $O\left(R^{-1 / 2}\right)$ :

$$
\left\{i\left(\omega-\alpha \Phi_{11}^{\prime}\right)\left(D^{2}-\alpha^{2}\right)+i \alpha \Phi_{0}^{\prime \prime \prime}+\frac{1}{R}\left(D^{4}+p \Phi_{0} D^{3}\right)\right\} \phi=0 \text {. }
$$

This equation has been derived by considering in turn the critical layer, the wall layer, and the rest of the boundary layer, ordering terms respectively in each of these, and by constructing a minimal composite equation containing all the terms which are $O\left(R^{-1 / 2}\right)$ or lower in any of the layers: the details are given in the following section. Equation (18) has the form of a modified OS equation. The differences between Eq. (18) and the OS equation (5) have been discussed in GN97, where it is shown that stability results from Eq. (18) are not qualitatively very different from those of more complete nonparallel equations, i.e., the lowest-order stability equation, in spite of being an o.d.e., contains substantially the effects due to the spatial development of the flow.

The approach used in GN97 permits us to derive a hierarchy of equations governing the stability of nonparallel flow. In GN97 the contribution due to the downstream nature of disturbance propagation was neglected; we now proceed to study this effect. In this process, we shall show that the next member in the hierarchy is a parabolic partial differential equation, correct to $O\left(R^{-2 / 3}\right)$, but still of lower order than the full nonparallel equation of GN95 or the PSE of BHS. The equation immediately higher in the hierarchy than the present equation would have to be $O\left(R^{-1}\right)$, which, it is argued, is inconsistent with mean flow data prescribed only up to $O(1)$.

\section{A HIERARCHY OF STABILITY EQUATIONS}

The objective of this section is to derive a sequence of composite stability equations of order lower than $R^{-1}$, each composite equation being capable of providing uniformly valid solutions to an appropriate order throughout the boundary layer. We begin by rewriting the full nonparallel equation (3) in the following form:

$$
\begin{aligned}
& {\left[\left(c-\Phi^{\prime}\right)\left(D^{2}-\alpha^{2}\right)+\Phi^{m}+\frac{1}{i \alpha R}\left\{D^{4}+p \Phi D^{3}\right.\right.} \\
& \left.\left.\quad+\left(-2 \alpha^{2}+\Phi^{\prime}\left(2 q-p-\frac{\partial}{\partial x}\right)\right) D^{2}+a_{1} D+a_{0}\right\}\right] 0=0,
\end{aligned}
$$

where $c$ is the phase velocity of the disturbance and $a_{0}$ and $a_{1}$ contain coefficients that can be derived from Eqs. (9) and (10). We define an expansion for the disturbance eigenfunction 0 as follows:

$$
\phi=\sum_{k} \epsilon^{k} \chi_{k}+\sum_{m} \epsilon^{m}(\log \epsilon) \lambda_{m}, \quad k=0,1,2, \cdots, \quad m=1.2, \cdots,
$$

where $\epsilon$ is a small parameter to be arrived at from the discussion below. The approach followed is to consider separately the bulk of the flow and the critical and wall layers, respectively, and to collect terms upto a given order into a composite stability equation in the manner described in GN97. Here the "bulk" of the flow includes the regions in the boundary layer above the critical layer as well as in between the critical and wall layers when these are distinct. In accordance with Eq. (20), the eigenfunction is expressed as an asymptotic expansion in each layer, respectively, as

$$
\begin{aligned}
\phi_{b}\left(y^{\prime}\right)= & \left.\chi_{b 0}\left(\eta_{b}\right)+\epsilon_{b} \chi_{b 1}\left(\eta_{b}\right)+\epsilon_{l}\right) \log \left(\epsilon_{b}\right) \lambda_{b 1}\left(\eta_{h}\right)+\cdots, \\
\phi_{c}(y)= & \chi_{c 0}\left(\eta_{c}\right)+\epsilon_{c} \chi_{c 1}\left(\eta_{c}\right)+\epsilon_{c} \log \left(\epsilon_{c}\right) \lambda_{c 1}\left(\eta_{c}\right) \\
& +\epsilon_{c}^{2} \chi_{c 2}\left(\eta_{c}\right)+\epsilon_{c}^{2} \log \left(\epsilon_{c}\right) \lambda_{c 2}\left(\eta_{c}\right)+\cdots, \\
\phi_{w 1}(y)= & \chi_{w 0}\left(\eta_{w}\right)+\epsilon_{w} \chi_{w 1}\left(\eta_{w 1}\right)+\epsilon_{w 1} \log \left(\epsilon_{1 w^{\prime}}\right) \lambda_{n 1}\left(\eta_{w^{\prime}}\right)+\cdots,
\end{aligned}
$$

with

$$
\eta_{b} \equiv y, \quad \eta_{c}=\frac{\left(y-y_{c}\right)}{\epsilon_{c}}, \quad \text { and } \quad \eta_{w}=\frac{y}{\epsilon_{w}} .
$$

$y_{c}$ is the critical height, at which the total mean velocity (including higher-order contributions) equals the phase velocity of the disturbance:

$$
c \equiv \frac{\omega}{a}=\Phi_{c}^{\prime} .
$$


The mean streamfunction $\Phi$ has already been written as the asymptotic expansion (13). The higher-order component of the mean flow in this equation, $\Phi_{1}$, contains contributions due to the displacement effects, surface curvature, etc. (see, e.g., Van $\left.D_{y k e^{7}}\right)$. In the flow over a semi-infinite wedge, $\Phi_{0}$ satisfies the Falkner-Skan equation, while $\Phi_{1}$ arises due to the displacement alone, and satisfies Eq. (17) of GN95. The lower-order component $\Phi_{0}$ can be expanded in the critical and wall layers, respectively, as

$$
\Phi_{0}=\Phi_{0 c}+\left(y-y_{c}\right) \Phi_{0 c}^{\prime}+\frac{\left(y-y_{c}\right)^{2}}{2} \Phi_{0 c}^{\prime \prime}+\cdots
$$

and

$$
\Phi_{0}=\frac{y^{2}}{2} \Phi_{0 w}^{n}+\cdots .
$$

Substituting Eqs. (21) and (13) into the full nonparallel Eq. (19), and equating terms of like order in $\epsilon_{b}$, we get the lowest-order equation for the outer layer, which is simply the Rayleigh equation

$$
\left(\omega-\alpha \Phi_{0}^{\prime}\right)\left(\chi_{b 0}^{\prime \prime}-\alpha^{2} \chi_{b 0}\right)+\alpha \Phi_{0}^{\prime \prime \prime} \chi_{b 0}=0
$$

From the corresponding equation of the next higher order we get

$$
\epsilon_{b}=(\alpha R)^{-1}
$$

In the critical layer, the lowest-order equation

$$
x_{c 0}^{(i v)}-i \eta_{c} \Phi_{0 c}^{\prime \prime} x_{c 0}^{\prime \prime}=0
$$

is obtained by setting

$$
\epsilon_{c^{\prime}}=(\alpha R)^{-1 / 3} \text {. }
$$

In Eq. (30), only the lower-order component of the mean flow appears. This is because, using Eq. (25), the factor ( $c$ - $\left.\Phi^{\prime}\right)$ in Eq. (19) can be expanded in the critical layer as

$$
c-\Phi^{\prime}=-\left(y-y_{c}\right) \Phi_{c}^{\prime \prime}-\frac{\left(y-y_{c}\right)^{2}}{2} \Phi_{c}^{\prime \prime \prime}-\cdots .
$$

Using Eqs. (24) and (13), and arranging in increasing powers of $\epsilon_{c}$, it is noticed that terms containing derivatives of the higher-order mean flow, $\Phi_{1}$, appear at higher orders in the expansion, and $\Phi^{\prime \prime}$ and $\Phi_{. . \prime}^{\prime \prime \prime}$ in the first two terms on the right hand side of Eq. (32) may be replaced, respectively, by $\Phi_{0 c}^{\prime \prime}$ and $\Phi_{0_{c}}^{\prime \prime \prime}$ up to the order considered.

In the wall layer, with

$$
\epsilon_{w}-T\left\{x P^{-1 / 2}\right.
$$

we get

$$
\chi_{w 0}^{(i v)}+i \Phi_{0 c}^{\prime} \chi_{w 0}^{\prime \prime}=0
$$

On examining Eq. (20) in the light of Eqs. (29), (31), and (33), it is seen that an appropriate choice for $\epsilon$ is $(\alpha R)^{-1 / 6}$. In Eqs. (21) and (23), the $X$ functions can be set to zero without any loss of generality, and Eq. (20) may be written as

$$
\begin{aligned}
\phi= & \chi_{0}+\epsilon^{2} \chi_{c 1}+\epsilon^{2} \log (\epsilon) \lambda_{c^{\prime} 1}+\epsilon^{3} \chi_{k 1}+\epsilon^{4} \chi_{c 2} \\
& +\epsilon^{4} \log (\epsilon) \lambda_{c 2}+O\left(R^{-1}\right) .
\end{aligned}
$$

Here, $\chi_{0}$ has contributions from all three layers. $x \backslash$ and $\chi_{5}$ in Eq. (20) are zero, while the primary contribution to $X_{2}$ and $\chi_{4}$ comes from the critical layer. The detailed discussion in the Appendix shows that the solutions for $\chi_{c}$ and $\lambda_{c}$ either vanish exponentially outside the critical layer or match with the inviscid solution. $\chi_{3}=\chi_{w^{\prime}}$ has an exponentially decaying behavior outside the wall layer (Drazin and $\operatorname{Reid}^{8}$ ), and so does not affect the matching.

The subsequent terms in Eq. (35) can be derived from Eq. (19) by the same procedure, and are solutions of the following equations:

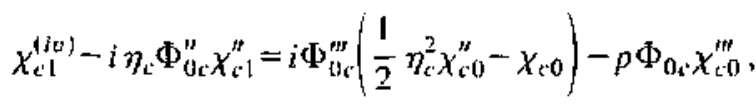

$\lambda_{i 1}^{(i v)}-i \eta_{c} \Phi_{0 c}^{\prime \prime} \lambda_{i 1}^{\prime \prime}=0$,

$\chi_{w 1}^{(i v)}+i \Phi_{0 c}^{\prime} \chi_{w 1}^{\prime \prime}=i \eta_{w} \Phi_{0 w}^{s}, \chi_{n, 0}^{\prime \prime}$,

$\chi_{c 2}^{(i v)}-i \eta_{c} \Phi_{b c}^{\prime \prime} \chi_{c 2}^{\prime \prime}=i \Phi_{0 c}^{m \prime}\left(\frac{1}{2} \eta_{c}^{2} \chi_{c 1}^{\prime \prime}-\chi_{c 1}\right)-p \Phi_{0 c} \chi_{c 1}^{\prime \prime \prime}-i \eta_{c}$

$$
\begin{aligned}
& \times\left[\Phi_{0_{c}}^{(j p)}+\alpha^{2} \Phi_{0 k^{\prime}}^{\prime \prime}\right] \chi_{c^{\prime} 0}-p \eta_{c} \Phi_{0 c}^{\prime} \chi_{c 0}^{\prime \prime \prime} \\
& +\left\{\frac{i \eta_{c}^{3} \Phi_{0 c}^{(i \omega)}}{6}+2 \alpha^{2}-(2 q-p) \Phi_{\omega c}^{\prime}\right\} \chi_{c+1}^{\prime \prime} \\
& +R \Phi_{0 c}^{\prime} \frac{\partial \chi_{\mathrm{c} 0}^{n}}{\partial x}
\end{aligned}
$$

$\lambda_{c 2}^{(i w)}-i \eta_{c} \Phi_{0,}^{\prime \prime} \lambda_{c 2}^{\prime \prime}=i \Phi_{0 c}^{\prime \prime \prime}\left(\frac{1}{2} \eta_{c}^{2} \lambda_{c-1}^{\prime \prime}-\lambda_{c^{\prime} 1}\right)-p \Phi_{0, c} \lambda_{c 1}^{\prime \prime \prime}$.

For reasons discussed earlier, the higher-order mean flow does not appear in any of the equations derived here.

From an examination of the above set of equations, it is evident [from the presence of the term containing the factor $p$ in Eq. (36)] that the lowest-order nonparallel effect is already present in the equation for $\chi_{c 1}$ which has an $O\left(R^{-1 / 3}\right)$ contribution. It is also apparent, from Eq. (39), that the streamwise derivative of the disturbance eigenfunction first appears in the coefficient of $\mathrm{e}^{4}$, i.e., at $O\left(R^{-2 / 3}\right)$.

We now return to the full nonparallel Eq. (3) and construct from it a "minimal" subset containing just those terms necessary for deriving equations for $\chi_{0}$ through $X 2$ and $\lambda_{1}$ and $\lambda_{2}$ in Eq. (20). This works out to be the lowest-order stability Eq. (18). Incidentally, the terms contained in Eq. (18) already include the equation for $\chi_{3}$, which makes the lowest-order stability equation correct to $O\left(R^{-1 / 2}\right)$. The next composite stability equation in this hierarchy would include $X 4$ and $\lambda_{4}$ and is given by

$$
\begin{gathered}
{\left[\left(\omega-\alpha \Phi_{0}^{\prime}\right)\left(D^{2}-\alpha^{2}\right\}+\alpha \Phi_{0}^{m}+\frac{1}{i R}\left\{D^{4}+p \Phi_{0} D^{3}\right.\right.} \\
\left.\left.+\left(-2 \alpha^{2}+\Phi_{0}^{\prime}\left(2 q-p-\frac{\partial}{\partial x}\right)\right) D^{2}\right\}\right] \phi=0 .
\end{gathered}
$$

It may be noticed that the last term in Eq. (41) contains the streamwise derivative of the disturbance amplitude, which was absent in the lowest-order Eq. (18), i.e., the effects of the parabolic nature of the flow on its stability first appear in this 
equation. It is therefore appropriate to call it the "Lowestorder Parabolic Stability Equation" (LOP equation). The boundary conditions are given by Eqs. (6) and (7).

It is important to note that the higher-order contributions to the mean flow, i.e., $\Phi_{1}$ and so on, do not affect stability upto the order considered. From Eq. (35) it is clear that the next stability equation in the hierarchy would be one correct to $O\left(R^{-1}\right)$ such as the full nonparallel equation of GN95. If we were to use such an equation, in order to be consistent we need to know $\Phi_{1}$ accurately. The LOP is thus the highestorder stability equation that is consistent with the lowestorder approximation to the mean flow in the boundary layer.

When the lowest-order parabolic equation is compared to the OS Eq. (5), it is noticed that the term $\alpha^{4} \phi$, which is present in the Orr-Sommerfeld equation, being $O\left(R^{-1}\right)$ or higher everywhere in the boundary layer, has to be neglected in this analysis. Instead, the term containing $D^{3} \phi$ and two additional terms containing $D^{2} \phi$ are now included. As discussed in GN97, the third derivative term is due to the advection of the disturbance vorticity, $\zeta_{l l}$, by the normal component of the mean velocity. The nonparallel component of the streamwise advection of $\xi_{d}$, on the other hand, gives rise to a new second derivative term as well as to the explicit parabolic term in Eq. (41). Equation (41) is a low-order subset of Eq. (3), which arises out of the vorticity equation for linear disturbances,

$$
\frac{D_{0} \zeta_{d}}{D t_{d}}+\frac{\partial^{3} \Phi_{d}}{\partial x_{d} \partial y_{d}^{2}} \hat{u}_{d}+\hat{v}_{d} \frac{\partial^{3} \Phi_{d}}{\partial y_{d}^{3}}-\nu \nabla_{d}^{2} \zeta_{d}=0 .
$$

Here $D_{0} / D t_{d}$ stands for the total derivative following the mean flow. The origin of each of the terms in Eq. (41) can be traced back to a corresponding term in Eq. (42) and the primitive equation for Eq. (41) may be derived to be

$$
\frac{D_{0} \xi_{d}}{D t_{d}}=-\hat{v}_{d} \frac{\partial^{3} \Phi_{d}}{\partial y_{d}^{3}}+\nu \frac{\partial^{3} \hat{a}_{d}}{\partial y_{d}^{3}}+2 v \frac{\partial^{3} a_{d}}{\partial x_{d}^{2} \partial y_{d}} .
$$

Equation (43) contains all nonparallel effects upto $O\left(R^{2} \quad 2 / 3\right)$; it is therefore sufficient to begin from Eq. (43) instead of (42) in order to obtain stability characteristics up to this order. This observation is relevant especially for nonsimilat flows where Eq. (41) will not hold. In comparison with the primitive equation for the lowest-order stability equation [Eq. (4.3) of GN97], it is seen that the only additional term in Eq. (43) is the last one, which represents streamwise diffusion of the dominant term in disturbance vorticity. In the LOP equation (as in the OS equation), this diffusion appears as an additional second derivative term $\left(-2 \alpha^{2} D^{2} \phi\right)$. Note that the last term in Eq. (43) is significant only at the critical layer, where the dominant contribution to $\zeta_{d}$ comes only from $\partial \hat{u}_{d} / \partial y_{d}$ : the other term $\partial \hat{v}_{d} / \partial x_{d}$ will be of higher order.

\section{RESULTS}

Neutral stability boundaries were computed using both the full nonparallel Eq. (3) and the LOP Eq. (41) for various heights in the boundary layer using the finite difference algorithm and solution procedure described in GN95. The main objective of this comparison is to enable an assessment of how accurate the present theory is in accounting for the

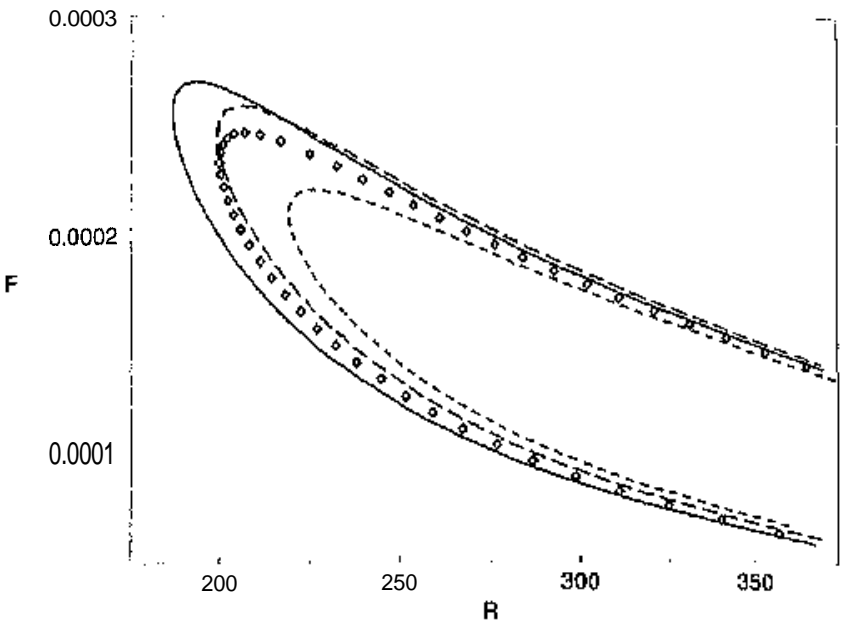

FIG. 1. Neulad stability boundaries al the imner maximum. $m=0$. Solicl lino: LOP equation; long dashes: "lull" monparalled theny: short datses: lowestorder theory; symbols: Orr-Sommerledd.

effects of flow nonparallelism; as allicaly pointed out, the "full nonparallel" theory [Eq. (6) of GN95] is to be considered consistent in any flow problem only if higher-order effects in boundary layer meall profiles vanish, The stability boundary at the inner maximum of the streamwise distulbance velocity, as predicted by different theories for the llow over a flat plate, is plotted in Fig. 1; here $F$ is the nonclimensional frequency parameter which is proportional to the dimensional frequency $\omega_{l l}$. (For a falkner-Skan mean flow with a pressure gradient partmeler $\mathrm{HI}$, we have $F$ $\equiv \omega / R^{(1-3 m) /(1+m)}$.) The results of BHS are practically indistinguishable from those of the full nonparallel Eq. (3) of GN95 and are therefore not indicated separately in the figure, Also not shown arc the DNS results of Fitsel and Konzelmann," with which the full nonparallel results are in excellent agreement.

It is well-known that in nonparallel flows assessment of stability depends on the path along which Ihe disturbance is monitored. At a given $R$, for example, the amplitude of $\|^{\prime}$ may decay along one path and grow along another, lisiscl and Konzelmann" and GN95 have shown that slabilily churacteristics are very sensitive to the normal distance from the wall of the monitoring location: thus the variation in the critical Reynolds number with height above the wall, for example, is certainly much larger in magnitude numerically (i.e., is of lower order in an asymptotic expausion) than $R^{\cdots 1}$. The dependence of stability on height is thus a good check for the performance of the LOP theory. We therchore compute stilbility boundaries at various heights in the boundary layer, and obtain from these two characleristic cuantitics at each height, namely the highest possible frequency $I_{\text {max }}$ at which instability is possible, and the critical Reynolds number $R_{\text {t' }}$.

The highest unstable frequency is plotted us a function of height in Fig. 2. It is evident that the LOP, in spile of being a much simpler equation than Eq. (3), is in close agreement with the predictions of the latter. As discussed in GN97, the conventional OS results shown in the figures are independent of height. (It is, however, possible to obtain height dependence from the OS by taking suitable directional 


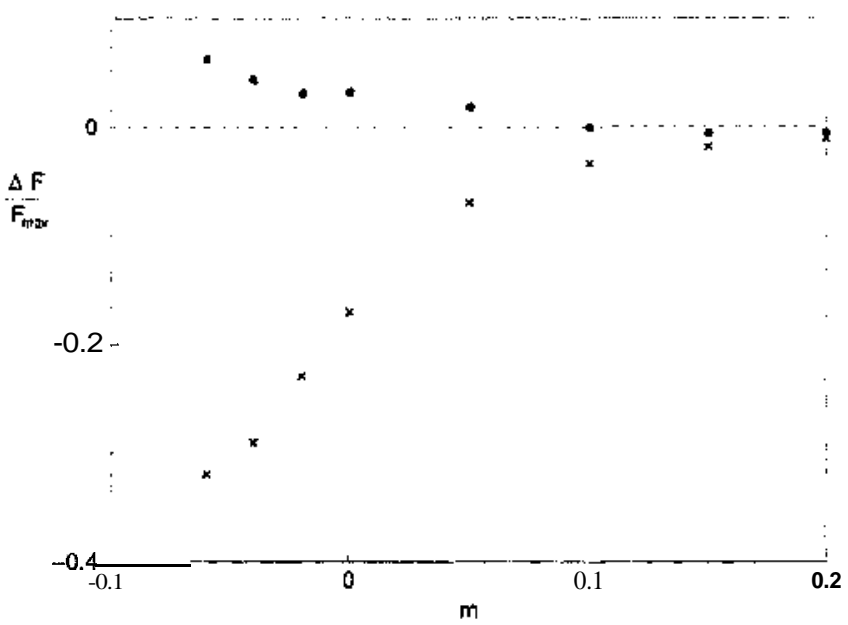

FIG. 6. Fractional error in highest unstable frequency. $\Delta F$ is the difference between the highest unstable frequency predicted by a low-order theory and that predicted by the full nonparallel theory for the same mean flow. Filled circles: LOP; crosses: lowest-order equation.

Reynolds number and the highest unstable frequency, are plotted against the pressure gradient: even in high adverse pressure gradients, the error is $\leqslant 10 \%$, while the error is much lower in favorable pressure gradients, presumably due to the high Reynolds numbers involved. As discussed in GN95, the streamwise derivative $\partial \phi / \partial x$ is arrived at by trial and error: in computations of full nonparallel solutions, numerical convergence is a problem, especially in adverse pressure gradients. As a result, some jitter was evident in the plots of neutral stability close to the critical Reynolds number, especially in sharply decelerating flows. However, for the LOP equation, convergence to the correct value is rapid for any $m$ and there is no jitter in the stability boundaries obtained.

In the results presented so far, the emphasis has been on the neutral stability boundary. This is important and is useful in assessing different stability theories, but, as shown in GN97, the stability loops at different heights can exhibit some rather peculiar features. A related but more important

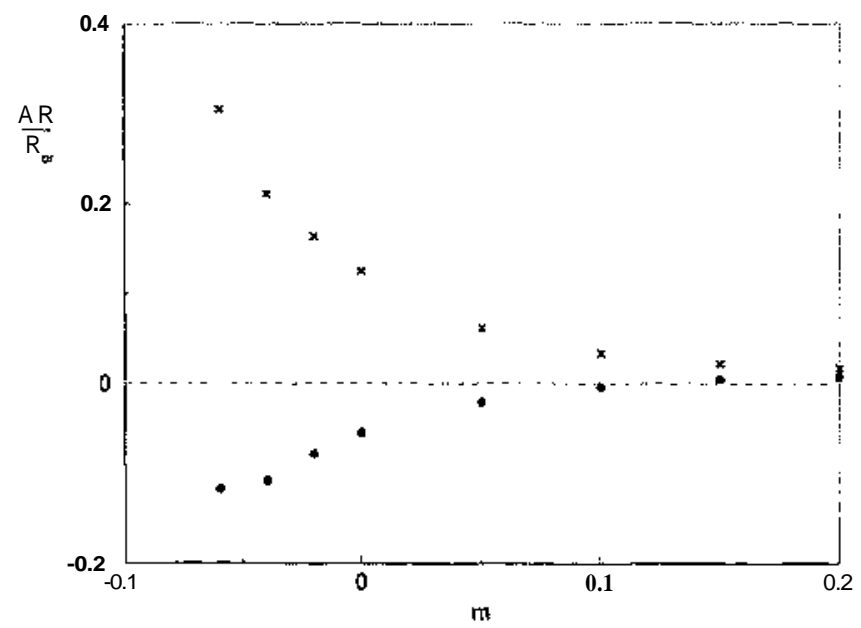

FIG. 7. Fractional error in critical Reynolds number. Filled circles: LOP; crosses: lowest-order equation.

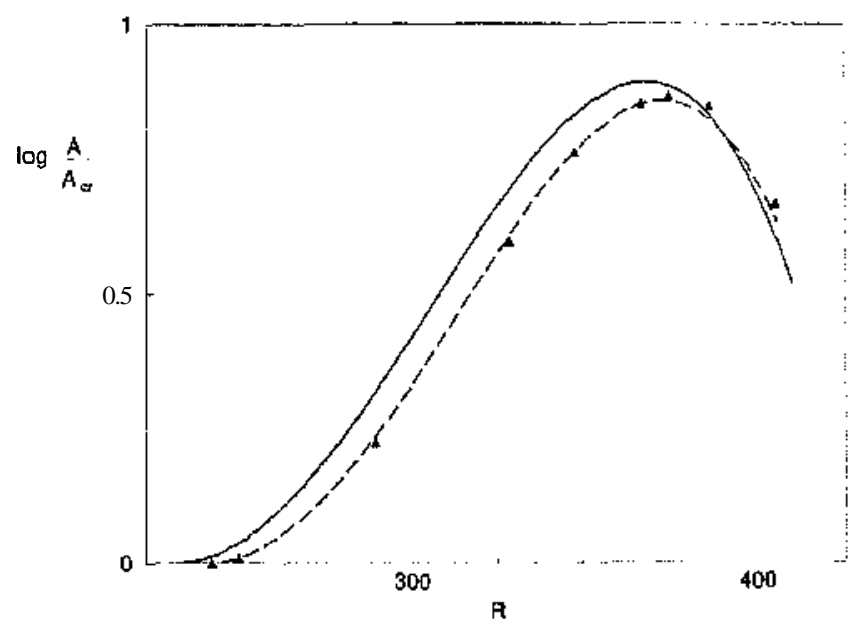

FIG. 8. Amplification factors at inner maximum for $F=1.4 d-4$ at $m=0$. Solid iine: LOP; long dashes: full nonparallel theory; symbols: Fase I and Konzelmann.

point is that, in nonparallel flows, there is a fundamental problem in apportioning streamwise valuation in disturbance amplitude between exponential growth [through the imaginary part of $a$ in Eq. (12)] and streamwise evolution [through the dependence on $x$ of $\phi(x, y)$ ].It is therefore perhaps more appropriate to examine directly the streamwise variation of the disturbance amplitude itself. Furthermore, for making predictions about the onset of transition to turbulence, it is again the amplitude of a given initial disturbance as a function of the streamwise location that is of primary relevance. In the familiar $e^{n}$ method (Smith and Gamberoni ${ }^{10}$ ), the envelope formed by plots of amplitudes corresponding to disturbances of different frequencies is monitored, and transition is predicted to take place at the location where its magnitude is $e^{\prime \prime}$ times the amplitude at the critical Reynolds number, i.e., where

$$
\log \left[\frac{A}{A_{\mathrm{er}}}\right]_{\max }=n
$$

$n$ being a prescribed number (usually taken as around 9).

Quantitative differences in $\log \left[A / A_{\mathrm{cr}}\right]$ obtained from different stability theories tend to be smaller than differences in stability boundaries, even in adverse pressure gradient flows, and the resulting $n$ factor varies little (GN95). This leads to the conclusion that lower-order theories, especially the LOP, should be sufficient for computations of $n$; at any rate, the greater accuracy of higher-order theories is of significance only when higher-order mean velocity profiles are available. Amplitudes computed using the full nonparallel Eq. (3) were shown by GN95 to be in excellent agreement with the results of the direct numerical simulations of Fasel and Konzelmann. ${ }^{9}$ Figure 8 shows the performance of the present theory in the computation of amplification factors at the inner maximum of the eigenfunction. The maximum discrepancy when compared to the full nonparallel solution is less than 0.1 , but this difference should be much smaller in the envelopes of disturbance amplitudes, i.e., in the $n$-factor. The LOP is in closer agreement with the full nonparallel results than the lowest-order results are. This is true even in adverse 


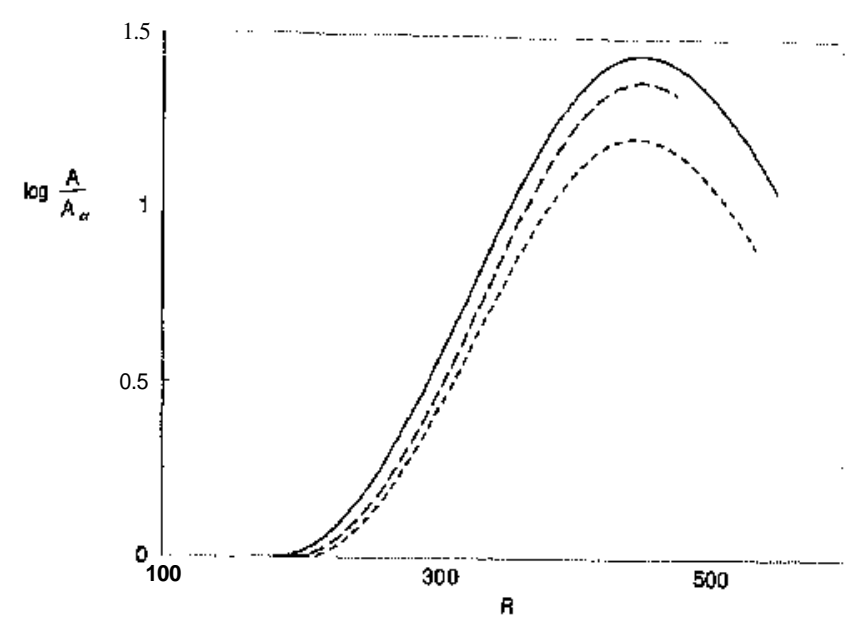

FIG. 9. Amplification factors at inner maximum for $F=5 . d-5$ at $m$ $=-0.04$. Solid me: LOP; long dashes: full nonparallel theory; short dashes: lowest-order equation.

pressure gradients, as shown in Fig. 9 for the Falkner-Skan flow at $m=-0.04$; again the difference in amplification factor is less than 0.1 .

\section{CONCLUSIONS}

We have derived and analyzed here the highest-order stability equation lower than the full nonparallel system of GN95, which is itself nominally correct to $O\left(R^{-1}\right)$. As has been pointed out in GN95 and GN97, an analysis including $O\left(R^{-1}\right)$ or higher-order terms would be justified only when mean velocity profiles from higher-order boundary-layer theory are used. To get a rational theory to these orders, one would have to expand the mean flow in the series given by Eq. (13); $\Phi_{1}$ would have to be included in the Rayleigh part of the stability equation to be consistent, as has been done in Eq. (3). However, we are aware of no stability calculations that include these higher-order effects, except for the example of the displacement thickness worked out in GN95. In most practical flows there are higher-order contributions to the mean flow, due for example to displacement thickness or surface curvature, but the mean flow data are often not available up to this level of accuracy. In such situations, use of the equation presented and analyzed in this paper is entirely legitimate. To get the full benefit of higher-order equations, like Eq. (6) of GN95 or the PSE, one would have to use higher-order boundary-layer theory.

The present paper has been confined to similar flows. It is important to stress that the extension of the LOP to nonsimilar flows is straightforward and will be addressed elsewhere. The approach followed is similar to that described in GN95, where a full nonparallel theory for "weakly" nonsimilar flows, such as in the boundary layers over airfoils, was developed and used to compute amplification factors over a typical airfoil.

\section{APPENDIX}

The two inviscid solutions of Eq. (28) for the outer layer have been given by Tollmien (Drazin and Reid ${ }^{8}$ ) as

$$
\begin{aligned}
& \left.\left[\chi_{h 0}\right]_{1}=\left(y-y_{c}\right) \mid\right]+\frac{\Phi_{b 0}^{\prime \prime}}{2 d_{0,}^{m}}(y-y) \\
& \left.+\frac{1}{6}\left[\frac{\Phi_{0}^{i c}}{\Phi_{0}^{n}}+u^{2}\right]\left(y-y_{c}\right)^{2}+\cdots\right) \text {. }
\end{aligned}
$$

and

$$
\begin{aligned}
{\left[\chi_{b 0}\right]_{2}=} & 1+\left[\frac{\Phi_{0 i c}^{i v}}{2 \Phi_{i c}^{\prime \prime}}-\frac{\Phi_{0 c}^{\prime \prime 2}}{\Phi_{0 i}^{\prime \prime 2}}+\frac{\alpha^{2}}{2}\right]\left(y-y_{c} y^{2}+\cdots\right. \\
& +\frac{\Phi_{0 c}^{\prime \prime \prime}}{\Phi_{0 i}^{\prime \prime}} \log \left(y-y_{\mathrm{c}}\right)\left[\chi_{b 0}\right]_{1} .
\end{aligned}
$$

It is shown below that the equations for the critical and wall layers obtained using the LOP Eq. (41) match with the above equations at large $\eta_{i}$ and $\eta_{u^{\prime}}$, respectively. It is observed that the terms which appear due to the nonparallel nature of the flow, i.e., those which are not taken into account in the classical theory, while being significant in the critical and wall layers, do not affect the matching with the outer layer. The critical layer equation (30) is the same as that in classical theory, so its solutions are well known. Two solutions of Eq. (30) may be obtained by setting $\chi_{r(1)}^{\prime \prime}=0$, which are $\left[\chi_{c} 0\right]_{1} \sim \eta_{c}$ and $\left[\chi_{c 0}\right]_{2}=1$. At large $\eta_{c}$, these two solutions match with the leading terms in Eqs. (A1) and (A2) of the inviscid solutions $\left[\chi_{b}\right]_{1}$ and $\left[\chi_{b}\right]_{2}$, respectively. For $\chi_{c-1}^{\prime \prime}$ $\neq 0$, Eq. (30) corresponds to Airy's equation for $\chi_{c 0}^{\prime \prime}$, which can be solved at large $\eta_{c}$ to give two solutions. One of these $\left(\left[\chi_{c 0}\right]_{4}\right)$ grows exponentially with $\eta_{c}$, which does not satisfy the matching condition. It is hence discarded, leaving the other solution with the following rapid decay outside the critical layer:

$$
\left[\chi_{c 0}\right]_{3} \sim \eta_{c}^{-5 / 4} \exp \left[-\frac{2}{3}\left(i \Phi_{0 c}^{\prime \prime}\right)^{-1 / 2} \eta_{c}^{3 / 2}\right]
$$

At the next order in the critical layer is Eq. (36), in which $\chi_{c 0}$ appears on the right hand side. Outside the critical layer, i.e., at large $\eta_{c},\left[\chi_{c 0}\right]_{3} \rightarrow 0$, and the solution $\left[\chi_{c: 1}\right]_{3}$ of Eq. (36) would again be one that rapidly decays outside the critical layer, therefore not affecting the matching. Thus the relevant particular integrals of Eq. (36) as $\eta_{c} \rightarrow x$ are those obtained by substituting $\left[\chi_{c 0}\right]_{1}$ and $\left[\chi_{c 0}\right]_{2}$ on ther ${ }^{\text {hi }}{ }^{\text {ht hand }}$ side of Eq. (36). In both of these cases, $\chi_{c 0}^{\prime \prime}=0$ and hence the nonparallel term $p \Phi_{0 c} \chi_{c(0)}^{\prime \prime \prime}$, although significant within the critical layer does not affect the matching conditions at large $\eta_{c}$. The solution at large $\eta_{c}$ is therefore the same as that from classical theory, i.e.,

$$
\left[\chi_{c i}\right]_{1}\left(\eta_{c} \rightarrow \infty\right) \sim \frac{\Phi_{0 c}^{\prime \prime \prime}}{2 \Phi_{0 c}^{n}} \eta_{c}^{2}
$$

which matches with the second term in Eq. (A1) and

$$
\left[\chi_{c 1}\right]_{2}\left(\eta_{c} \rightarrow \infty\right)=\frac{\Phi_{0 c}^{\prime \prime \prime}}{\Phi_{0 c}^{\prime \prime}} \eta_{c} \log \eta_{c},
$$

which, together with

$$
\left[\lambda_{c t}\right]_{2}\left(\eta_{c} \rightarrow \infty\right)=\frac{\Phi_{0 c}^{\prime \prime}}{\Phi_{0 c}^{n}} \eta_{t^{\prime}},
$$


matches the term containing $\left(y-y_{c}\right) \log \left(y-y_{c}\right)$ in Eq. (A2). By the same argument, nonparallel terms in Eq. (39) are not important at large $\eta_{c}$ and the matching obeys classical theory. The two solutions relevant for matching with the outer layer are

$$
\left[X_{c}, 2\right]_{1}\left(\eta_{c} \rightarrow \infty\right) \sim \frac{1}{6}\left[\frac{\Phi_{0 c}^{i c}}{\Phi_{\nu_{c}}^{i t}}+\alpha^{2}\right] \eta_{c}^{3}
$$

and

$$
\begin{aligned}
{\left[\chi_{c-\tau}\right]_{2}\left(\eta_{c^{\prime}} \rightarrow x\right)=} & {\left[\frac{\Phi_{0 c}^{i v}}{2 \Phi_{0 c}^{\prime \prime}}+\frac{\alpha^{2}}{2}-\left(\frac{\Phi_{0 c}^{m}}{\Phi_{0 c}^{\prime \prime}}\right)^{2}\right] \eta_{c}^{2} } \\
& +\left(\frac{\Phi_{0 c}^{\prime \prime \prime}}{2 \Phi_{0 c}^{\prime \prime}}\right)^{2} \eta_{c}^{2} \log \eta_{c}
\end{aligned}
$$

Equation (40) for $\lambda_{c 2}$ is thesame as Eq. (36), whose solution has the same form as Eq. (A4):

$$
\left[\lambda_{c 2}\right]_{2}\left(\eta_{c}-x\right)=\frac{\Phi_{0 c}^{m}}{2 \Phi_{0 c}^{m}} \eta_{c}^{2}
$$

It can easily be verified that these solutions match with the third terms of Eqs. (A1) and (A2) respectively. For the wall layer, all solutions of Eqs. (34) and (38) decay outside the wall layer and do not appear in the matching process.

${ }^{1} \mathrm{M}$. Gaster, "On the effects of boundary layer growth on flow stability," J. Fluid Mech. 66, 465 (1974).

${ }^{2}$ F. P. Bertolotti, Th. Herbert, and P. R. Spalart, "Linear and nonlinear stability of the Blasius boundary layer," J. Fluid Mech. 242, 441 (1992).

${ }^{3}$ R. Govindarajan and R. Narasimha, "Stability of spatially developing boundary layers in pressure gradients," J. Fluid Mech. 300, 117 (1995).

${ }^{4} \mathrm{R}$. Govindarajan and R. Narasimha, "A low-order theory for stability of nonparallel boundary-layer flows," Proc. R. Soc. London, Ser. A $\mathbf{4 5 3}$, 2537 (1997).

${ }^{5}$ M. Simen, S. Hein, F. Bertolotti, V. Wendt, A. Hanif, and D. Henningson, "Nonlocal and local instability analysis of hypersonic flows," in LemminarTurbulent Transition, edited by R. Kobayashi (Springer-Verlag, New York, 1995), pp. 147-154.

${ }^{6} \mathrm{~F}$. T. Smith, "On the nonparallel flow stability of the Blasius boundary layer," Proc. R. Soc. London, Ser. A 366, 91 (1979).

${ }^{7}$ M. Van Dyke, "Higher-order boundary-layer theory," Annu. Rev. Fluid Mech. 1, 265 (1969).

${ }^{8}$ P. G. Drazin and W. H. Reid, Hydrodynanic Stability (Cambridge University Press, Cambridge, 1981).

${ }^{9}$ H. F. Fasel and U. Konzelmann, "Nonparallel stability of a Mall plate boundary layer using the complete Navier-Stokes equations," I. Fluid Mech. 221, 311 (1990).

${ }^{10}$ A. M. O. Smith and N. Gamberoni, "Transition, pressure gradient and stability theory," Douglas Aircraft Company, Rep. ES26388 (1956). 